\title{
Systematic excited state studies of reversibly switchable fluorescent proteins
}

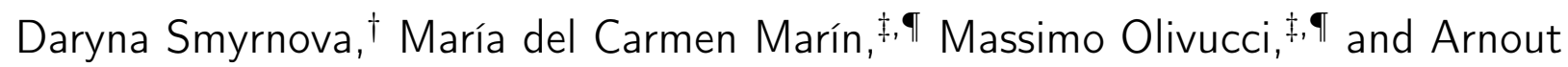
Ceulemans ${ }^{*, \dagger}$

$\dagger$ †uantum Chemistry and Physical Chemistry Division, Department of Chemistry, $K U$ Leuven, Celestijnenlaan 200F, 3001 Heverlee, Belgium

$\ddagger$ Department of Biotechnology, Chemistry e Pharmacy, Universitá di Siena, via A. Moro 2, I-53100 Siena, Italy

IDepartment of Chemistry, Bowling Green State University, Bowling Green, OH 43403, $U S A$

E-mail: arnout.ceulemans@kuleuven.be,daryna.smyrnova@kuleuven.be 


\section{Introduction}

While basic photophysical mechanisms ${ }^{1,2}$ allow for the wide range of applications of the Green Fluorescent Protein (GFP) and its closely related systems, ${ }^{3-5}$ fluorescent proteins (FPs) featuring more complex mechanisms have been discovered over the years providing new application opportunities. ${ }^{6-9}$ One example is the discovery of Reversibly Switchable Fluorescent Proteins (RSFPs), ${ }^{10-12}$ which have become an indispensable tools in high-resolution microscopy ${ }^{13,14}$ and whose photophysics and photochemistry are still being investigated and optimized. ${ }^{15,16}$

RSFPs have also been the target of several theoretical studies which have contributed, for instance, to the understanding of the factors controlling their fluorescence ${ }^{2}$ and isomerization ${ }^{17}$ quantum yields as well as their color-tuning. ${ }^{18}$ However, in spite of such efforts, the answer to fundamental questions such as why some FPs are photo-switching while others are only florescent, is still incomplete. Such questions are intimately related to the understanding of how, in general, a change in the protein environment affects the properties of the chromophore. More specifically, it is not clear how few $\beta$-barrel mutations may shift the wavelength of the absorption maxima by $40 \mathrm{~nm},{ }^{19,20}$ what are the distinct roles played by the chromophore structure and by its environment in such process and, most importantly, how does each amino acid replacements contribute to the observed changes. To provide an answer to the above questions, different groups have investigated the impact of the FP environment on the wavelength of the absorption maximum ${ }^{21}$ and other observables. ${ }^{22,23}$ For instance, a study on a set of green, orange and red FPs by Hasegawa et al. ${ }^{22}$ has reported on blue-shifting effects caused by electrostatic changes in the environment (i.e. amino acid residues with different charge distributions). Another study by List et al. ${ }^{24}$ has identified the role of the environment in one- and two-photon absorption of DsRed. Other models were build to explain pressure and temperature effects ${ }^{25}$ and spectral tuning in GFP related systems. ${ }^{26}$ Furthermore, the mapping of the potential energy surfaces of the isolated anionic GFP chromophore by Martin et $\mathrm{al}^{27}$ and Polyakov et $\mathrm{al}^{28}$ have provided mechanistic 
information on possible excited state isomerization paths.

One target of the theoretical/modeling studies mentioned above is to uncover principles useful for the engineering of improved or completely novel tools for fluorescence microscopy or other fluorescence applications. However, it has to be reckoned that the achievement of an understanding solid enough to allow for the design of novel FPs, requires the investigation of sets of computer models (from now on, simply, models) constructed using exactly the same protocol. Indeed, such models are expected to avoid the inconsistencies generated when using different methodologies and building workflows, thus providing more accurate information on the trends of photophysical properties. This is highly desirable if one expects to learn how such trends originate from specific amino acid replacements eventually obtaining effective engineering principles. For this reason, here we present the results of the investigation of a set of consistent models of RSFPs featuring the same chromophore and differing by a minimal number of amino acid replacements (i.e. mutations). Using such set we will explore the changes in excitation energies and, in part, characterize the excited state potential energy surface connecting the absorption, emission and radiationless deactivation regions involved in the isomerization driven photo-switching mechanism. Our investigation is focused on Dronpa $^{10,29}$ and derived systems. Due to its pioneering role as a member of RSFPs, ${ }^{7,30}$ many Dronpa mutants were developed and characterized. ${ }^{19}$ For our study we have selected a limited set of Dronpa mutants featuring the same chromophore and featuring amino acid replacements located in both the $\beta$-sheets and/or $\alpha$-helix (see Table 1 ). 


\begin{tabular}{|c|c|c|c|}
\hline Protein & $\begin{array}{l}\text { Mutation, relative to } \\
\text { Dronpa }\end{array}$ & $\begin{array}{l}\text { Absorption, nm } \\
(\mathrm{kcal} / \mathrm{mol})\end{array}$ & $\begin{array}{l}\text { Emission, nm } \\
(\mathrm{kcal} / \mathrm{mol})\end{array}$ \\
\hline Dronpa $^{29}$ & - & $503(56.7)$ & $522(54.7)$ \\
\hline rsFastLime $^{31}$ & V157G & $496(57.5)$ & $518(55.1)$ \\
\hline rsKame $^{32}$ & V157L & $503(56.7)$ & $515(55.4)$ \\
\hline \multirow[t]{4}{*}{ Padron0.9 $9^{33,34}$} & $\mathrm{~T} 59 \mathrm{M}+\mathrm{V} 60 \mathrm{~A}+$ & $504(56.6)$ & $510(56.0)$ \\
\hline & $\mathrm{N} 94 \mathrm{I}+\mathrm{P} 141 \mathrm{~L}+$ & & \\
\hline & $\mathrm{G} 155 \mathrm{~S}+\mathrm{V} 157 \mathrm{G}+$ & & \\
\hline & M159 + F190S & & \\
\hline \multirow[t]{3}{*}{ bsDronpa $^{19}$} & $\mathrm{~A} 69 \mathrm{~T}+\mathrm{D} 112 \mathrm{~N}+$ & $460(62.0)$ & $504(56.6)$ \\
\hline & $\mathrm{G} 155 \mathrm{~S}+\mathrm{V} 157 \mathrm{G}+$ & & \\
\hline & $\mathrm{M} 159 \mathrm{C}+\mathrm{F} 173 \mathrm{C}$ & & \\
\hline
\end{tabular}

Table 1: Effect of mutations on the observed wavelength of the absorption and emission maxima (also expressed in terms of vertical excitation energies) of Dronpa-like systems. Only the most relevant mutations are indicated for Padron0.9. For bsDronpa six mutations are shown, which were also used to model it on the basis of the Dronpa crystal structure.

Each RFSP in Table 1 has been selected for a specific reason. Two Dronpa single-point mutants rsKame ${ }^{32}$ and rsFastLime ${ }^{19,31}$ were chosen for their minimal structural and spectral differences with wild-type Dronpa but with large differences in photo-switching speed. In order to study broader spectral variations, we have chosen Padron0.9 ${ }^{34}$ and bsDronpa. ${ }^{19}$ Both proteins conserve the chromophore structure and feature only a few mutations in the protein matrix. Padron0.9 is a negatively-switchable counterpart of Dronpa with, notably, a wavelength of the emission maxima slightly blue-shifted in comparison to Dronpa. In contrast, bsDronpa is a blue-shifted mutant of Dronpa with a remarkably large Stokes shift $(43 \mathrm{~nm})$. Previous studies on FPs with large Stokes shifts ${ }^{20,35,36}$ have shown that large shifts are the result of a flexible hydrogen bond network (HBN) where, possibly, ground and/or 
excited state surface shows HBN variations. Thus, here our focus is to see if it is possible to obtain a bsDronpa model capable to correctly reproduce the observed spectral trend of the RSFP set without indulge into a detailed conformational analysis of bsDronpa HBN fluctuations.

We emphasize that the selected RSFPs differ by a maximum of eight mutations and originate from the same protein thus limiting the differences in the chromophore environment and, most importantly, in the global protein structure. This is an important choice in the context of the present study. In fact, since we are ultimately interested in the computational designing of functional mutants using relatively simple protein models and building protocols (see below), the conservation of the original protein structure is highly desirable. On the other hand, the more amino acid replacements are considered, the highest is the chance of unpredictable structural deformation of the original Dronpa structure and, thus, of constructing unrealistic models. For the same reason the selected RSFPs not only feature a common chromophore $\pi$-system including phenol and imidazoline rings, but also the same chromophore side-chains which have been shown to affect the $\alpha$-helix flexibility and the adaptation of the chromophore to its environment. ${ }^{29,37,38}$

Different studies have shown that the details of the model building protocol affect the calculated excitation energy values and, most importantly, the variation calculated when comparing different FPs. ${ }^{21,39,40}$ For this reason, and as anticipated above, we use the same quantum chemical based protocol for building the selected RSFP models. This is an adapted version of the Automatic Rhodopsin Modelling (ARM) protocol developed by Melaccio et $\mathrm{al}^{40}$ yielding quantum mechanics/molecular mechanics (QM/MM) models. As stressed in the original reference, such protocol is not designed to produce the most accurate QM/MM models possible, but basic, gas-phase and computationally fast models aimed to the rationalization and prediction of trends between sequence variability and function. Accordingly, the QM/MM models used here are based on the complete-active-space self-consistent-field (CASSCF) treatment of the QM subsystem with an MM (protein and solvent) subsystem 
described using the conventional Amber force field. As detailed in Section 4, the QM/MM model building initiates starting from extensive molecular dynamics (MD) simulation to select suitable guess structures. Such guess structures constitute the basis for the construction of QM/MM models that, after geometry optimization, are used to compute the excited and ground state energies at the multiconfigurational second order perturbation (CASPT2) level of theory. The final excited state QM/MM structures of each protein are then employed for investigating the structure of the excited state reaction path including the conical intersection regions where decay to the ground state occurs. 


\section{Results}

2.1 Reproducing the experimental absorption and emission energies

\section{Absorption and Emission maxima, $\mathrm{kcal} / \mathrm{mol}$ Calculated vs. Experimental}

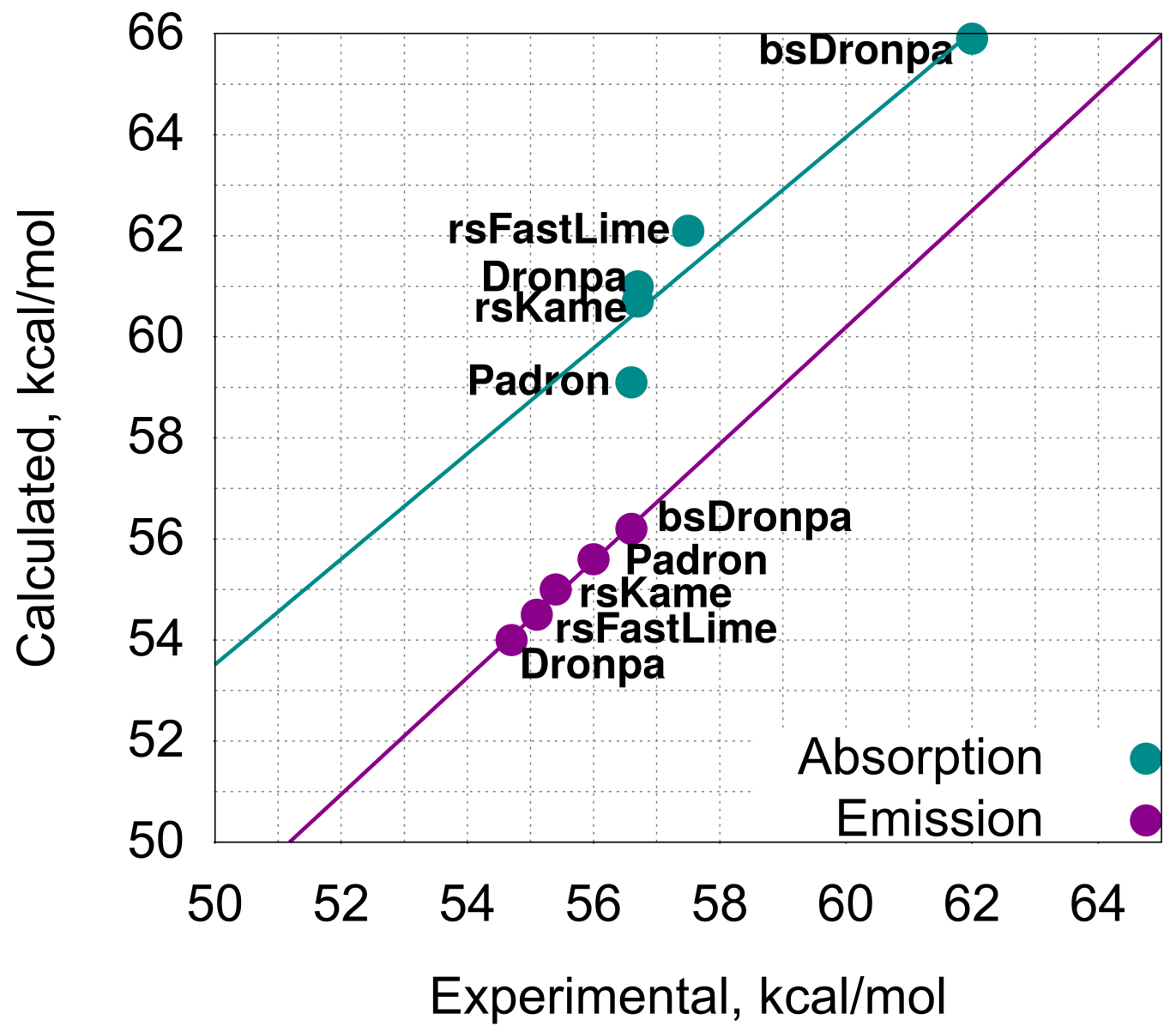

Figure 1: Comparison of the calculated and experimental absorption and emission energies for the selected set of five RSFPs.

The energies corresponding to the computed wavelength of the absorption and emission maxima (expressed, as described in Section 4 , in terms of average $\mathrm{S}_{1}-\mathrm{S}_{0}$ vertical energy differences of ten sampled structures. $\mathrm{S}_{1}$ is the potential energy of the first singlet excited 
state and $\mathrm{S}_{0}$ is the corresponding ground state energy), from now on called absorption and emission energies respectively, are shown in Figure 1. The trends of the observed (i.e. experimental) emission and absorption energies are reproduced. The standard deviation of the calculated emission energies with respect to the corresponding experimental values is ca. $1.5 \mathrm{kcal} / \mathrm{mol}$. The calculated absorption energies are instead more blue-shifted with a standard deviation of ca. $3.5 \mathrm{kcal} / \mathrm{mol}$ standard.

\section{Emission vs. Absorption maxima, kcal $/ \mathrm{mol}$ in protein and vacuum}

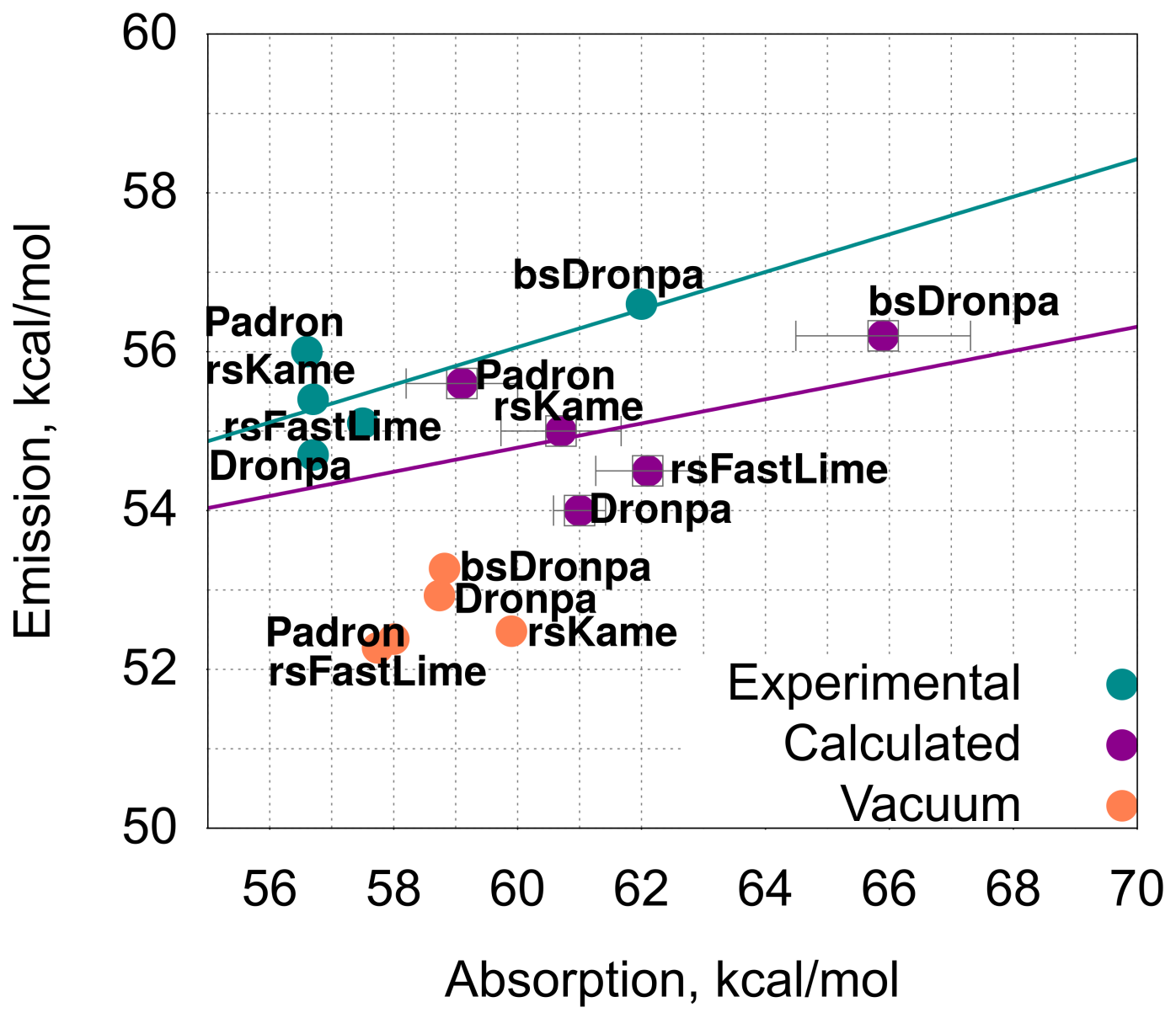

Figure 2: Comparison of the chromophore absorption and emission energies in the protein and in vacuum.

As it can be seen from both Figure 1 and Figure 2, mutations can significantly affect 
the absorption energy, while the emission energy appears to be less sensitive (i.e. featuring a reduced spread in values). In principle, this behavior can be associated to the protein induced changes of the chromophore geometry (e.g. of its conformation) and/or of the environment electrostatics (e.g. point charges of the surrounding residues) along the absorption and emission series. To assess the influence of the geometrical changes, we have recalculated the absorption and emission energies after extracting the optimized chromophore from the protein (orange points in the Figure 2). These "vacuum" chromophore results show reduced changes in the absorption and also emission energies, leading to the conclusion that the variation in the protein electrostatic caused by the mutations dominates the variations observed along the series. Figure 2) is also reporting on the possible existence of a proportionality relationship between emission and absorption energies. The plot shows that there is no clear correlation between absorption and emission energies. In other words, a higher absorption wavelength does not imply a higher emission maximum.

\subsection{Chromophore charge redistribution}

The sensitivity to the changes in the protein cavity electrostatics described above needs to be explained. For instance, one want to explain why such effect leads to a $6 \mathrm{kcal} / \mathrm{mol}$ absorption energy difference between Padron0.9 and bsDronpa compared to the only $2 \mathrm{kcal} / \mathrm{mol}$ maximum difference in emission energy between Dronpa and bsDronpa. In order to do so, we have looked at the chromophore charge redistribution (i.e. the atomic charge difference between $\mathrm{S}_{1}$ and $\mathrm{S}_{0}$ ) occurring after either excitation or emission and, essentially, due to an

electron density change along the chromophore $\pi$-system. The results are reported in Figure 3. 


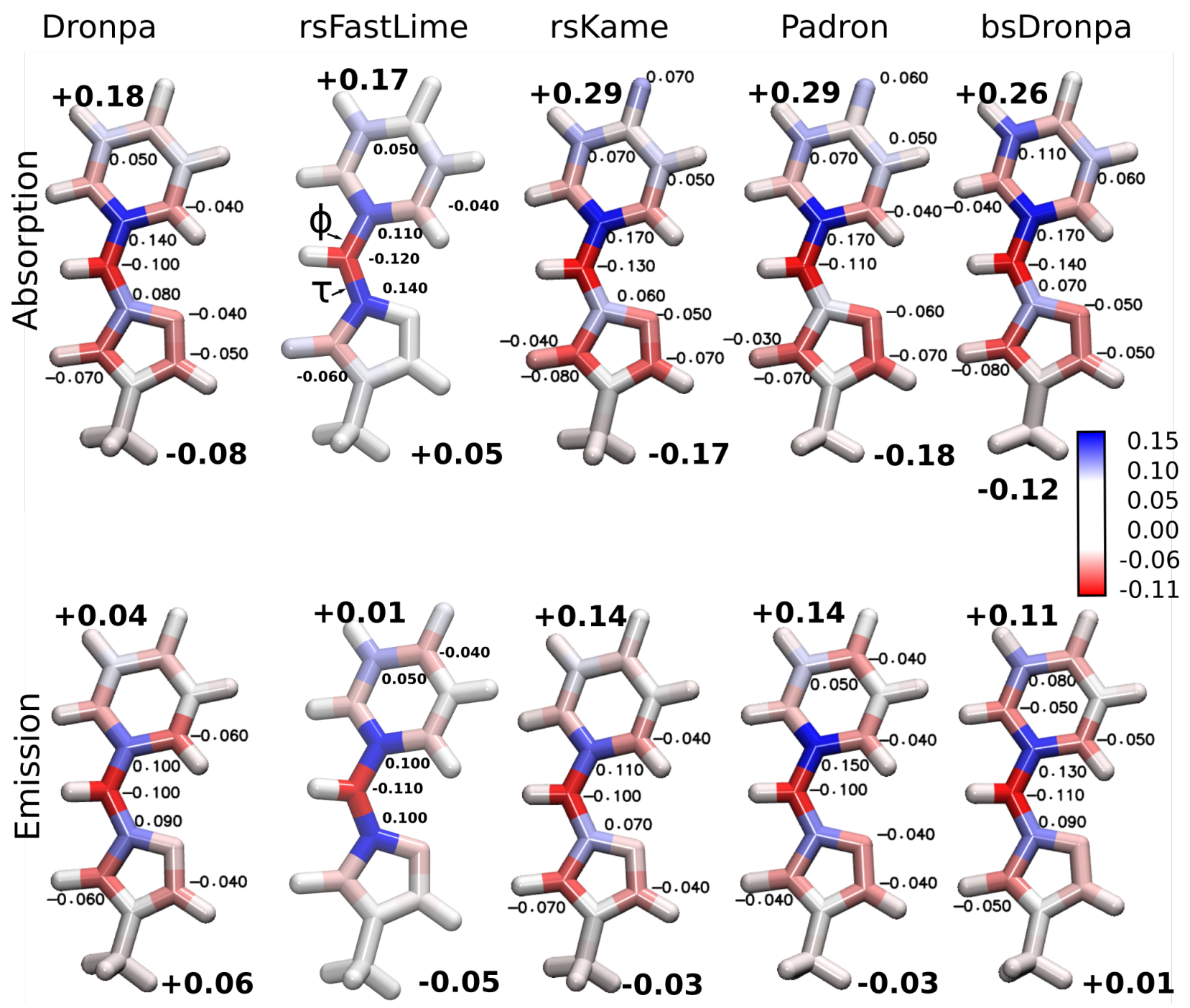

Figure 3: Comparison of the charge redistribution in the chromophore during absorption and emission. The images are color coded with the color bar shown on the right. Values are indicated next to the atoms for which the charge difference exceeds 0.04. Hydrogen charges are summed into the heavy atoms. The net charge difference between $S_{1}$ and $S_{0}$ on phenol and imidazole parts of the chromophore is indicated in bold next to respective structures.

The figure shows that the excited state charge transfer pattern (the blue and red colors in the figure correspond to positive and negative charge differences between the excited state and the ground state respectively) is very similar for all proteins regardless the number of mutations which characterize them. These patterns are in line with the findings of Filippi et al. ${ }^{39}$ for the anionic form of GFP. Accordingly, during the absorption the negative charge is transferred from the phenol ring to the imidazoline ring and the bridging atoms of the 
single-double bond bridge. As a consequence, a positive charge develops on the phenol ring. Similar charge transfer patterns were observed in the corresponding chromophore in vacuum (see Figure S2). From a quantitative point of view, the charge transfer is most pronounced in rsKame, Padron and bsDronpa than in Dronpa e rsFastLime where the charge redistribution is mostly located on the $\mathrm{C}-\mathrm{CH}-\mathrm{C}$ bridge atoms.

The described charge redistribution can also be interpreted on the basis of the relationship between the electronic and the geometrical structure of the chromophore (i.e. mainly focusing on the $\tau$-bond and $\phi$-bond displayed in Figure 3 ). In $\mathrm{S}_{0}$ the $\tau$-bond has a prevalent double character while the $\phi$-bond has a single bond character (benzenoid resonance structure). The computed bridge $S_{1}$ charge redistribution, which shows a net increase in positive charge, corresponds to an electronic delocalization and weakening of the $\pi$-bonding over $\tau$ and $\phi$ which would facilitate the double-bond isomerization (and shift to a quinoid structure).

Although, the charge redistribution pattern looks similar for absorption and emission, the net charge transfer from phenol to imidazoline is, as expected when looking at the different sensitivities described above, more pronounced during the absorption. The difference is especially pronounced in rsKame, Padron and bsDronpa, where the charge redistribution is almost two times smaller in the case of emission. Remarkably, no difference was observed in vacuum. Hence the higher magnitude of the spread of values during the absorption must be induced by the protein environment.

\subsection{Chromophore relaxation and $\mathrm{HBN}$ changes}

The geometrical parameters of the chromophore which display the largest changes during the protein excited state relaxation, are reported in Figure S2. Consistently with the charge redistribution and changes in bond lengths discussed above, the results indicate that the chromophore backbone is preparing for the isomerization as indicated by the increase in $\phi$ and $\tau$ dihedral angles. In fact, the double bond bridge elongation, associated with the increase in single bond character, prompts a noticeable out-of-plane conformational twisting 
mainly around $\tau$ (by 21 degrees in the case of Dronpa). The charge redistribution has also an impact on the HBN geometry. For instance, in Dronpa, as well as in rsFastLime and rsKame, the charge transfer accompanying light absorption and then excited state relaxation (i.e. the transfer from the phenol ring into the imidazoline ring) result in a strengthening (a decrease in length) of the H-bonds between the imidazoline ring and the Arg 89 and nearby water molecule. Concurrently, the increase in positive charge of the chromophore phenol induces weakening of the H-bonds with the nearby water molecules and/or Ser 142. These changes occur together with a chromophore induced H-bond length modification leading to a slight Glu 144 - His 193 H-bond shortening and Glu 144 - Arg 64 bond elongation. Since Padron and bsDronpa have a slightly different protein environment, the H-bonds look different, but follow the same chromophore induced changes.

The effects of the above changes may be described as that of Arg 89 and Arg 64 acting as a pair of positively charged tweezers pulling the chromophore via a negatively charged imidazoline ring. In this way more space is given to the phenol ring preparing for the isomerization. It has been shown earlier by Morozov et al. ${ }^{41}$ that a lower number of H-bonds with the chromophore, facilitates the isomerization. The described H-bond length variations seem to support such conclusion. Also, in our previous studies ${ }^{38}$ we have shown that during the thermal isomerization a whole $\alpha$-helix moves with the chromophore thus creating a space necessary for the isomerization. Here the same type of motion is observed on the excited state. A recent outstanding experimental study by Coquelle et al. ${ }^{42}$ has also demonstrated that the chromophore isomerization is accompanied by an alpha-helix movement.

\subsection{Impact of the protein residues on the absorption and emission energies}

In order to document the influence of the individual amino acids on the absorption and emission energies, we have recomputed these quantities after turning off the charges of the most relevant residues. The results of such calculation are reported in Figure S3. The results 
shows that the absorption and emission energies are very sensitive to the Arg 64 and Arg 89 charges. The importance of these residues can be attributed to their strong interaction with the chromophore through the HBN (see above). His 193 also shows a large effect as it is involved in the $\pi$-stacking interaction with the phenol ring of the chromophore. In fact, as reported by $\mathrm{Li}$ et al, ${ }^{43}$ the H193T mutation shows how the absence of this interaction induces the internal conversion and leads to fluorescence loss. The interaction of the chromophore with Tyr 116 is often overlooked but our calculations indicate its importance. To our knowledge there is no experimental data published for Dronpa Tyr 116 mutants, however Y116Q and Y116N mutations in pcDronpa (a green-to-red photoconvertible mutant of Dronpa) ${ }^{29}$ lead to an increased photo-conversion quantum yield and higher pKa of the protein. The blue-shifting effect of Arg 89 clearly manifests itself in bsDronpa, where it loses interaction with the chromophore. Although, for a definitive answer an X-ray structure of bsDronpa must be awaited, we anticipate that a large Stokes shift occurs due to Arg 89 flexibility already discussed above. When its interaction with the chromophore is switched off (for Dronpa - rsKame - rsFastLime - Padron) and in practice (bsDronpa) a blue-shift in absorption is observed both in theory and experimentally.

\subsection{Conical intersections and excited state isomerization paths}

Several studies have shown that conical intersections (CIs) act as "bottlenecks" for lightinduced isomerization reactions. More specifically, CIs provide efficient radiationless decay channels located about halfway along the double bond isomerization coordinate. Thus the properties of the CI region may have an impact on the photo-switching of RSFPs when this is based on an isomerization process. One common mechanistic hypothesis, is that the energy of a CI relative to the excited state minimum (i.e. the "fluorescent state" of the protein) would provide information on the probability of accessing it and, therefore, on the isomerization speed. For this reason, we now focus on the characterization of the lowest lying CI's (also indicated as MECI) for the selected RSFPs. The fact that we already 
have consistent information on the excited state minimum (min-S $\mathrm{S}_{1}$ ) of each RSFP, and that the QM/MM models of the CIs can be built using exactly the same protocol, makes the geometrical and energy comparison meaningful.

A first target of our CI computations is to verify the existence of an inverse proportionality between computed MECI energies and observed isomerization speeds (i.e. as mentioned above). This is important because, according to the current mechanistic view, the isomerization speed would be inversely proportional to the fluorescence quantum yield (as seen in the Table S1) and, therefore, the MECI energy would provide information on such critical quantity. On the other hand, this possibility is based on a simplistic view of the $\mathrm{S}_{1}$ potential energy profile along the isomerization coordinate which would be monotonically decreasing or increasing when moving from min- $\mathrm{S}_{1}$ the MECI is reached. However, factors such as the presence of exited state energy barriers controlling the access to the CI region as well the specific topography of the CI region may play and important role and make the excited state dynamics more complex. To locate MECIs located at the end of the excited state isomerization path of RSFPs we have used the CI optimization method available in Molcas. ${ }^{44}$ Consistently with the ground state and min-S $\mathrm{S}_{1}$ calculations, CI optimizations were carried out at CASSCF $(12,11) / 6-31 \mathrm{G}^{*}$ level while the CI energies were computed performing single point CASPT2 calculation at the optimized structures. Further details can be found in Section 4 .

The results of our calculations are reported in Figure 4. For the triad rsFastLime Dronpa - rsKame, the MECI energies are inversely proportional to the observed isomerization quantum yields and photo-switching speed. Namely, rsFastLime is the fastest switch and its MECI has the lowest energy (only $3.7 \mathrm{kcal} / \mathrm{mol}$ above the corresponding excited state minimum), then follows Dronpa $(4.0 \mathrm{kcal} / \mathrm{mol}$ above the corresponding excited state minimum) and rsKame - the slowest switch - has the highest MECI energy of all three (7.9 kcal/mol above the corresponding excited state minimum). On the basis of the results for these three RSFPs one could conclude that the MECI energy indeed determines the 
photo-switching speed. Following this conclusion one would then expect that the MECI energies of Padron0.9 and bsDronpa, which are the fastest switches of the series, be even lower than rsFastLime energy and perhaps even negative (i.e. with a MECI located lower in energy than min- $\left.\mathrm{S}_{1}\right)$. However, following numerous attempts, we had to conclude that, using our models, the MECI energies of Padron0.9 and bsDronpa stayed at $22.1 \mathrm{kcal} / \mathrm{mol}$ and $16.4 \mathrm{kcal} / \mathrm{mol}$ above the emitting $\min -\mathrm{S}_{1}$ respectively. These results suggest that, the isomerization process guiding the photo-switching occurs according to more complex mechanisms possibly transition states connecting the min- $\mathrm{S}_{1}$ to the MECI region and/or efficient decay prompted by the specific topography of the MECI region and not by its energy value. 


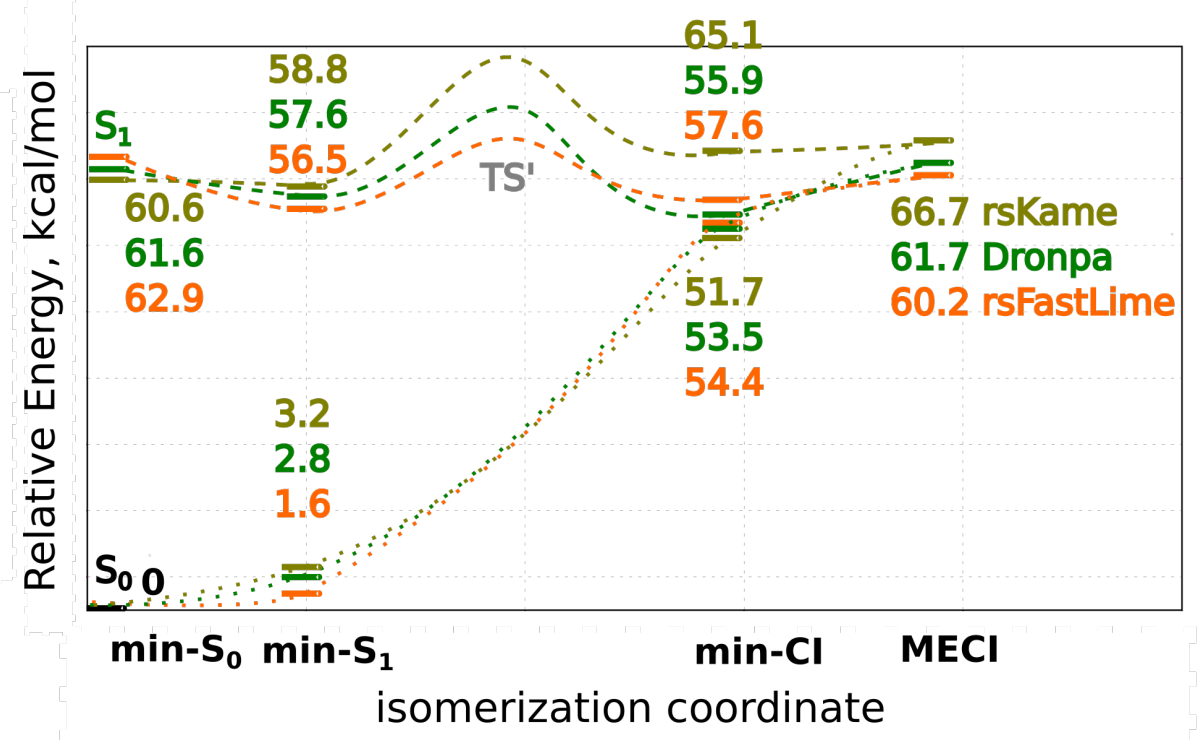

(a)

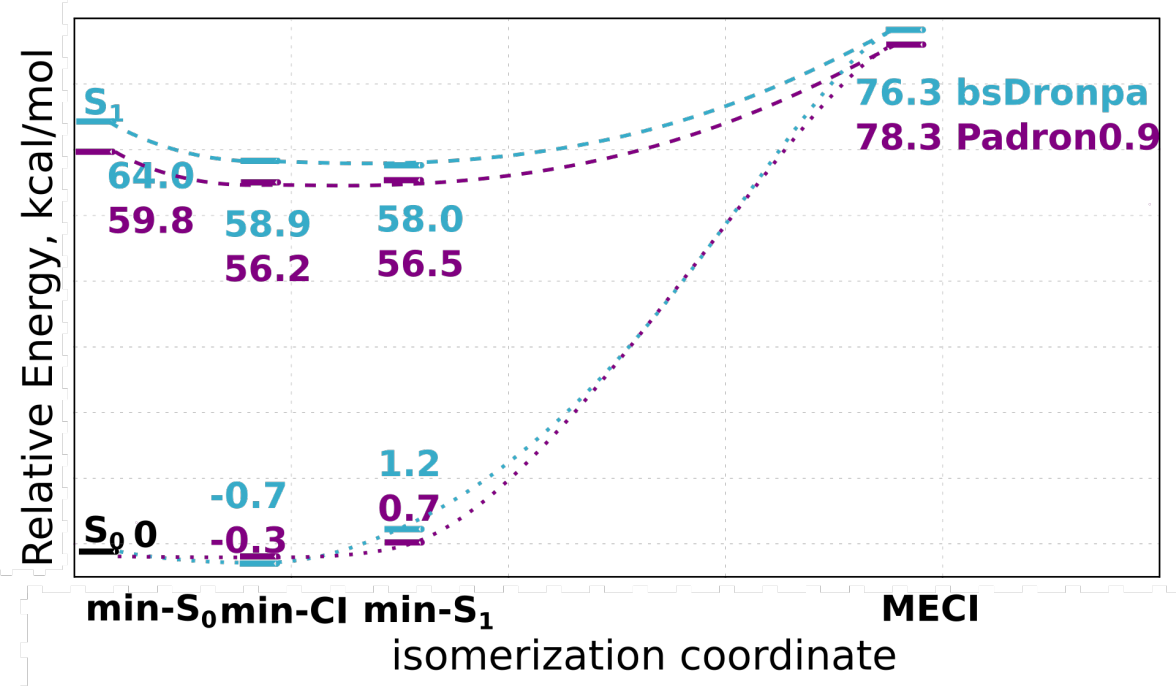

(b)

Figure 4: Potential energy surface of the chromophore isomerization in a) rsKame - Dronpa rsFastLime and b) bsDronpa - Padron. The energy values are indicated in the corresponding color. Dashed and dotted lines provide qualitative representation of the ground and excited state surfaces respectively. The energy maxima indicate the hypothetical positions of TS regions. The $\mathrm{S}_{1}$ isomerization coordinate connects the $\mathrm{FC}$ region (min- $\mathrm{S}_{0}$ ) to the fluorescent state $\left(\min -\mathrm{S}_{1}\right)$ and this to the MECI. See text for a description of min-CI.

In order to better characterize the min- $\mathrm{S}_{1}$ to the MECI region we have initially considered the possibility to locate the transition states mentioned above. However, the systematic 
search and optimization of transition states using our current QM/MM methods is difficult and time consuming. Its degree of success depends critically on the quality of the second derivative matrix which cannot be computed analytically. For this reason, and after a few failed attempt, here we simply try to find evidence for the existence of such transition states without actually locating them. To do so, we have looked for local highly twisted minima by starting a geometry optimization from the optimized MECI geometries. Since the optimization method employed is locally convergent, one expects to get qualitatively different optimization outcomes in the presence or absence of a transition state. In fact, if a transition state exists that separate the min- $\mathrm{S}_{1}$ and MECI regions, one expects to find either a novel energy minima located very close to the CI points (min-CI) or an energy minima corresponding to the MECI point itself. From a topological perspective, the existence of such highly twisted min-CI point would imply the existence of an energy maximum (actually, first order saddle point) corresponding to a transition states) separating min-CI and min- $\mathrm{S}_{1}$ (and therefore the CI and min- $\left.\mathrm{S}_{1}\right)$. In contrast, if such geometry optimization leads to a structure corresponding to the previously located min- $\mathrm{S}_{1}$, such a transition state cannot exist.

For rsFastLime - Dronpa - rsKame min-CI points were found in the vicinity of the corresponding CI points (see Figure 4a) with energies of 1.1, 2.2 and $6.3 \mathrm{kcal} / \mathrm{mol}$ above min- $\mathrm{S}_{1}$ respectively. However, the energy minima located for Padron0.9 and bsDronpa were identified at substantially the same energy and geometry of min- $\mathrm{S}_{1}$ (indicated as min- $\mathrm{S}_{1}{ }^{\prime}$ ). These results indicate that transition states exist for rsFastLime - Dronpa - rsKame but not for Padron0.9 and bsDronpa. RMSD comparison can be found in the Table S2 and superimposed chromophore structures from FC (i.e. the min-S $\mathrm{S}_{0}$ structure) and MECI are shown in the Figure S4. In Figure S4 the optimized MECI are shown in magenta and the minCI are shown in orange. Consistently with the discussion reported above, in the rsFastLime - Dronpa - rsKame case the min-CI and MECI structures are barely distinguishable. In conclusion, when considering all findings, it can be deduced that the CI region of rsFastLime - Dronpa -rsKame is separated by an energy barrier from the fluorescent state region, while 
no such barrier exist for Padron0.9 and bsDronpa .

\section{Conclusions}

After selecting a suitable set of RSFPs we have shown that, consistent (i.e. produced with exactly the same protocol) QM/MM models of the ground and excited state minima of each member of the set reproduce the observed trends in absorption and emission energies. This suggests that our models provide a viable tool for rational fluorescent protein design even if the absolute values of such energies appear to be few kcal/mol blue-shifted.

An analysis of the ground and excited states characterizing the absorption and emission processes, provided information on the properties of the selected photo-switches. For instance, charge redistribution analysis and individual amino acid contributions have ultimately shown that the absorption energy is more sensitive of the emission energy to mutations. Since, both the absorption and emission energies appear to be dominated by changes in the $\mathrm{S}_{1}$ rather than $\mathrm{S}_{0}$ energy, we conclude that such a behavior is dominated by the sensitivity of the excited state electronic structure (i.e. that of a charge transfer state) to the amino acid replacement. Then one can propose an explanation for the higher sensitivity of the absorption energy. During the electronic excitation the system (i.e. during the light absorption process) the molecule is in equilibrium in the ground and not in the excited state, while during the emission it is in equilibrium in the excited state. It is therefore likely that the environmental changes perturb the system more during the absorption and less during the emission. bsDronpa with its remarkable Stokes shift (i.e. the $5.4 \mathrm{kcal} / \mathrm{mol}$ difference between absorption and emission energy), which is significantly higher than that of the rest

of the selected RSFP, serves as an example of the impact of the flexibility of the protein environment. In our models the major difference between bsDronpa from the other proteins is the absence of a H-bond between Arg 89 and the imidazole ring of the chromophore (our calculations clearly show that Arg 89 contributes to a blue shift in absorption). This lack 
of H-bonding would allow a larger stabilization of the excited state after relaxation due to a decreased rigidity of the chromophore surroundings.

Above we have also investigated how the MECI stability with respect to the fluorescent state could control the photo-switching speed. Our results suggest that such control indeed occurs for Dronpa - rsFastLime - rsKame. However, it has to be noted that the computed proportionality between CI stability and photo-switching speed may actually reflect the proportionality between the heights of the barriers controlling access to the CI regions. These barriers would be determined by corresponding transition states (i.e. the energy maxima along the dashed energy profiles of Figure 4a) which are higher than the MECI.

The existence of transition states with energies higher than the MECI would explain the apparent discrepancy between the CIs of Dronpa - rsFastLime - rsKame and between the much higher MECIs of Padron0.9 and bsDronpa which do not show evidence for the existence of transition states. For these fast switching RSFPs, we have attempted to locate MECIs by: i) starting from an isomerized (trans) chromophore, ii) using as guess optimization structures the CIs from Dronpa - rsFastLime - rsKame and iii) starting the CI optimiziation from interpolated structures connecting the min-S $\mathrm{S}_{1}$ and MECI geometries. All attempts have led to structures with very similar energies to the originally located MECIs. Both Padron0.9 and bsDronpa have lower $\mathrm{QY}_{\text {fluo }}$, which suggests that system can proceed from $\mathrm{FC}$ region to a CI faster, thus resulting in a higher isomerization speed. Hence, the hypothesis that the CI height above the excited state minima provide information on the barrier controlling the isomerization, appears to be incorrect.

In conclusion, the RSFP decay process must be more complex than commonly assumed and requires further investigations to be understood. Possibly, the characterization of the sloped MECI topography (the five located CI are all sloped rather than peaked), such as it's degree of tilting may have an impact to the photo-switching speed. On the other hand, the use of excited state classical trajectory calculations could provide more useful mechanistic details. In fact, the simulation of the excited state population dynamics at room temperature 
starting from an equilibrated ensemble of protein structures and velocities, should increase our understanding on the photo-switching process. This should be particularly relevant in the case of bsDronpa which features an higher conformational flexibility. Another possibility is to consider coupled proton-transfer and isomerization mechanisms which may take place in protein environment. For instance, in Padron0.9, and consecutively in bsDronpa as well, His 193 is neutral, while it is protonated in Dronpa - rsFastLime - rsKame. Elucidating the origin of this difference in protonation states might shed light onto the high-lying MECI's in Padron0.9 and bsDronpa. And finally, recent results by Park et al. ${ }^{45}$ suggest that the dynamical correlation may play an important role in structure optimization as well, hence turning to a CASPT2 MECI optimization is also considered.

\section{Computational Methods}

\section{Molecular Dynamics (MD) and Replica-Exchange MD (REMD)}

The first step in the selection of suitable guess structures for successive QM/MM model building is an extensive conformational sampling of all five proteins. This has been performed via MD simulations performed using Gromacs 5.0.1 ${ }^{46}$ package and the Amber99sb-ILDN forcefield. ${ }^{47} \mathrm{~A}$ detailed description of the employed MD protocol for Dronpa, rsFastLime

and rsKame can be found in our previous work. ${ }^{38,48} \mathrm{~A}$ similar scheme has been adopted for Padron0.9 and bsDronpa. For Dronpa and Padron0.9, the crystallographic structures of their bright states were used ( $2 Z 10^{49}$ and $3 Z \mathrm{UJ}^{50}$ respectively) to initiate the simulation. Instead, the initial models of rsKame, rsFastLime and bsDronpa were obtained via point mutations of the Dronpa crystallographic structure. Again, the list of mutations differentiating Dronpa from the rest of the proteins is given in Table 1.

In all cases $\mathrm{PROPKA}^{51}$ predictions were used to determine the protonation states of the titratable residues. In the case of Padron0.9 we have adopted the protonation scheme proposed by Brakemann et al., ${ }^{34}$ where GLU 144 is protonated and HIS 193 is neutral with 
a proton placed on $\mathrm{N} \epsilon$. Crystal waters present in the X-ray structure were conserved. Then proteins were solved in a $80.5 \times 80.5 \times 80.5 \AA^{3}$ TIP3P water box and neutralized by $\mathrm{Na}^{+}$and $\mathrm{Cl}^{-}$counterions added at the concentration of $0.15 \mathrm{M}$. The prepared systems were minimized and equilibrated at $300 \mathrm{~K}$ temperature and 1 bar pressure for 100ps. Long-range interactions were included using Particle Mesh Ewald method, cut-off distance was set $13.5 \AA$. Since a good quality crystal structure was available for Padron0.9, five 100 ns MD runs were enough for sufficient conformational sampling. A time step of 2 fs and SHAKE algorithm ${ }^{52}$ were employed. The temperature was controlled using velocity rescaling with a stochastic term. ${ }^{53}$

The bsDronpa model has been constructed by introduction of 6 point-mutations (listed in the Table 1) to Dronpa structure. In order to assure a thorough conformational search a Temperature Replica-Exchange MD (T-REMD) simulation was performed. Twenty structures were assigned temperature values from $300 \mathrm{~K}$ to $330 \mathrm{~K}$ which were obtained using the

temperature generator server for REMD-simulations. ${ }^{54}$ Equilibration was performed at each of 20 temperatures following the same procedure as described for MD simulation. Then 50 ns runs were performed for each replica thus leading to $1 \mu \mathrm{s}$ cumulative production run. The resulting exchange probability was $\approx 20 \%$ with exchange attempts occurring every 2 ps.

GROMACS utilities were used to perform cluster analysis for each of five proteins. As a results the median structure (the most representative for each simulation) was determined. Then these structures were used as input structures for QM/MM simulations.

The $Q M / M M$ calculations

The MOLCAS/Tinker interface ${ }^{55}$ was used to perform QM/MM calculations. MOLCAS $7.8^{55}$ was used to describe the QM part and Tinker $5.1^{56}$ for the MM part. In preparation of the QM/MM models the Automated Rhodopsin Modelling (ARM) ${ }^{40}$ approach was used. This tool has proved to be a robust protocol for simulations of rhodopsin-like photoreceptors. Certainly there is structural difference between Dronpa-like fluorescent proteins and rhodopsin-like photoreceptors. However similarity in their photobiological characteristics allows for an employment of the same approach. The QM/MM model was constructed on the 
basis of a median structure obtained from the classical MD simulations described above. Proteins were re-centered in the water box, then a sphere of $30 \AA$ radius from the chromophore was defined. Since no periodic boundary conditions are employed during the QM/MM simulations this approach allows to save computing time without losing the environment effect. QM/MM model can be divided into: the MM subsystem formed by the environment and the chromophore cavity parts and the QM subsystem formed by the chromophore. As an illustrative example, the model constructed for Dronpa is shown in the Figure 5.

In all cases, the input structure used for $\mathrm{QM} / \mathrm{MM}$ model was subject to $10 \times 1 \mathrm{~ns} \mathrm{MD}$, where the environment was kept frozen and cavity atoms flexible. Then, 10 random frames were picked up from the resulting trajectories and used for consecutive QM/MM calculations. Thus, for each protein, 10 independent QM/MM models were prepared.
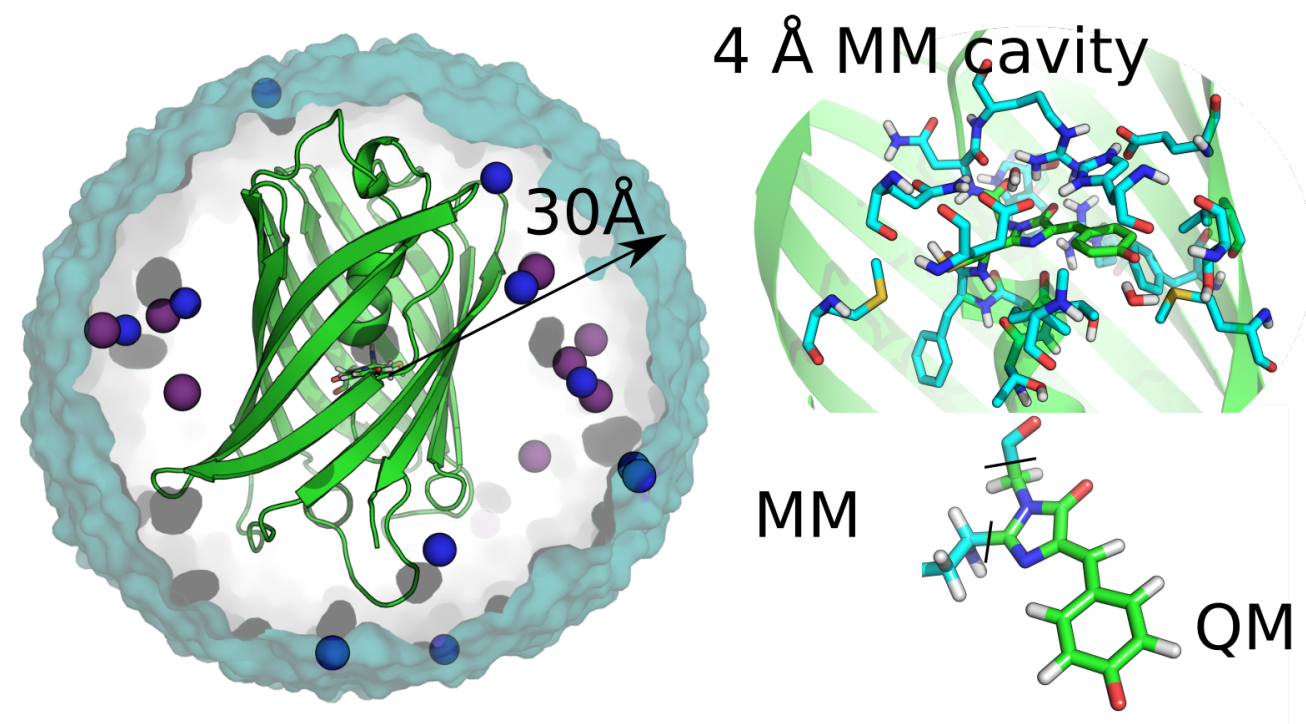

Figure 5: Full system is shown on the left (protein shown as cartoon, $\mathrm{Na}+$ and $\mathrm{Cl}$ - ions as spheres (blue and violet respectively), dark cyan surface indicates a $30 \AA$ water sphere and chromophore is shown as sticks). A $4 \AA$ radius cavity around the chromophore was defined and comprised of $\mathrm{X}$ residues (cyan) including the chromophore (green). All this residues were flexible during the optimization. The water molecules present in the crystal structure were maintained and included in the cavity. Chromophore was included in the QM region.

The atoms belonging to the environment part were fixed. The $4 \AA$ radius cavity was defined around and including the chromophore. Residues in the cavity part were free to 
relax. The QM part comprised of the chromophore's $\pi$-system with link atoms ${ }^{57}$ placed between carbon atoms (shown in the Figure 5). The link atom positions were restrained by applying the Morokuma scheme. ${ }^{58}$ The charges of the frontier atoms were set to 0 and the residual fractional charge redistributed along the rest of the MM atoms incorporated in the "chromophore residues" according to atomic mass and insuring a total -1 integer charge. In all calculations MM atoms were described with Amber94 ${ }^{59}$ force field.

The QM/MM model building started with a first step were an initial structure optimised at the HF/3-21G/MM level was obtained. Then a single-point CASSCF $(12,11) / 6-31 \mathrm{G}^{*} / \mathrm{MM}$ calculation was performed in order to define/verify the correct active space orbitals. These corresponded to all $\pi$-orbitals with occupancies not close to 0 or 2 . The resulting orbitals were then used as input for the $\mathrm{CASSCF}(12,11) / 6-31 \mathrm{G}^{*} / \mathrm{MM}$ geometry optimization. In order to obtain the correct energies we then performed CASPT2(12,11)/6-31G*/MM 3single root single point calculation is performed, taking a 3-roots State Average (SA) ${ }^{60}$ CASSCF $(12,11) / 6-31 G^{*} / \mathrm{MM}$ wavefunction as reference. As a result 10 vertical excitation energies $\left(\Delta \mathrm{E}_{\mathrm{S} 1-\mathrm{S} 0}\right)$ were computed. Average over 10 values for each protein was taken as a reference for comparison to the experimental values. To calculate the standard deviation from the experimental values we have used the standard formula given in Eq.1

$$
\sigma=\sqrt{\frac{1}{N} \sum_{i=1}^{N}\left(x_{i}-x_{e x p}\right)^{2}}
$$

where $\mathrm{N}=10$ and $\mathrm{x}_{\exp }$ is an experimental value.

Charge re-distribution shown in the Figure 3 was calculated as a difference between the Mulliken charges in the ground and excited states. Hydrogen charges were summed up to the heavy atoms. To calculate the effect of each amino acid on absorption and emission maxima the most representative structures for each protein were used for single-point CASPT2(12,11)/6-31G*/MM 3-single root calculation. Charges of atoms corresponding to each amino acid were set 0 in the Tinker key-file. 


\section{Acknowledgement}

Financial support from the Flemish Government through the KU Leuven concerted action scheme is gratefully acknowledged. The computational resources and services used in this work were provided by the VSC (Flemish Supercomputer Center), funded by the Hercules Foundation and the Flemish Government.

\section{Supporting Information Available}

Following information can be found in the Supporting Information: computational details, This material is available free of charge via the Internet at http://pubs.acs.org/.

\section{References}

(1) Okabe, M.; Ikawa, M.; Kominami, K.; Nakanishi, T.; Nishimune, Y. 'Green Mice' as a Source of Ubiquitous Green Cells. FEBS Lett. 1997, 407, 313-319.

(2) Lelimousin, M.; Noirclerc-Savoye, M.; Lazareno-Saez, C.; Paetzold, B.; Le Vot, S.; Chazal, R.; Macheboeuf, P.; Field, M. J.; Bourgeois, D.; Royant, A. Intrinsic Dynamics in ECFP and Cerulean Control Fluorescence Quantum Yield. Biochemistry (Mosc.) 2009, 48, 10038-10046.

(3) Grynkiewicz, G.; Poenie, M.; Tsien, R. Y. A New Generation of Ca2+ Indicators with Greatly Improved Fluorescence Properties. J. Biol. Chem. 1985, 260, 3440-3450.

(4) Shaner, N. C.; Campbell, R. E.; Steinbach, P. A.; Giepmans, B. N. G.; Palmer, A. E.; Tsien, R. Y. Improved Monomeric Red, Orange and Yellow Fluorescent Proteins Derived from Discosoma Sp. Red Fluorescent Protein. Nat Biotech 2004, 22, 1567-1572.

(5) Shimomura, O.; Johnson, F. H.; Saiga, Y. Extraction, Purification and Properties of 
Aequorin, a Bioluminescent Protein from the Luminous Hydromedusan,Aequorea. $J$. Cell. Comp. Physiol. 1962, 59, 223-239.

(6) Fernández-Suárez, M.; Ting, A. Y. Fluorescent Probes for Super-Resolution Imaging in Living Cells. Nat Rev Mol Cell Biol 2008, 9, 929-943.

(7) Dedecker, P.; De Schryver, F. C.; Hofkens, J. Fluorescent Proteins: Shine on, You Crazy Diamond. J. Am. Chem. Soc. 2013, 135, 2387-2402.

(8) Subach, F. V.; Verkhusha, V. V. Chromophore Transformations in Red Fluorescent Proteins. Chem. Rev. 2012, 112, 4308-4327.

(9) Kent, K. P.; Childs, W.; Boxer, S. G. Deconstructing Green Fluorescent Protein. J. Am. Chem. Soc. 2008, 130, 9664-9665.

(10) Habuchi, S.; Dedecker, P.; Hotta, J.-i.; Flors, C.; Ando, R.; Mizuno, H.; Miyawaki, A.; Hofkens, J. Photo-Induced Protonation/Deprotonation in the GFP-like Fluorescent Protein Dronpa: Mechanism Responsible for the Reversible Photoswitching. Photochem. Photobiol. Sci. 2006, 5, 567-576.

(11) Habuchi, S.; Ando, R.; Dedecker, P.; Verheijen, W.; Mizuno, H.; Miyawaki, A.; Hofkens, J. From The Cover: Reversible Single-Molecule Photoswitching in the GFPlike Fluorescent Protein Dronpa. Proc. Natl. Acad. Sci. 2005, 102, 9511-9516.

(12) Hofmann, M.; Eggeling, C.; Jakobs, S.; Hell, S. W. Breaking the Diffraction Barrier in Fluorescence Microscopy at Low Light Intensities by Using Reversibly Photoswitchable Proteins. Proc. Natl. Acad. Sci. 2005, 102, 17565-17569.

(13) Dedecker, P.; Hotta, J.-i.; Flors, C.; Sliwa, M.; Uji-i, H.; Roeffaers, M. B. J.; Ando, R.; Mizuno, H.; Miyawaki, A.; Hofkens, J. Subdiffraction Imaging through the Selective Donut-Mode Depletion of Thermally Stable Photoswitchable Fluorophores: Numerical 
Analysis and Application to the Fluorescent Protein Dronpa. J. Am. Chem. Soc. 2007, 129, 16132-16141.

(14) Vandenberg, W.; Leutenegger, M.; Lasser, T.; Hofkens, J.; Dedecker, P. DiffractionUnlimited Imaging: From Pretty Pictures to Hard Numbers. Cell Tissue Res. 2015, 360, 151-178.

(15) Bourgeois, D.; Adam, V. Reversible Photoswitching in Fluorescent Proteins: A Mechanistic View. IUBMB Life 2012, 64, 482-491.

(16) Acharya, A.; Bogdanov, A. M.; Grigorenko, B. L.; Bravaya, K. B.; Nemukhin, A. V.; Lukyanov, K. A.; Krylov, A. I. Photoinduced Chemistry in Fluorescent Proteins: Curse or Blessing? Chem. Rev. 2016, 117.

(17) Groenhof, G.; Bouxin-Cademartory, M.; Hess, B.; de Visser, S. P.; Berendsen, H. J. C.; Olivucci, M.; Mark, A. E.; Robb, M. A. Photoactivation of the Photoactive Yellow Protein: Why Photon Absorption Triggers a Trans-to-Cis Isomerization of the Chromophore in the Protein. J. Am. Chem. Soc. 2004, 126, 4228-4233.

(18) Shcherbakova, D. M.; Subach, O. M.; Verkhusha, V. V. Red Fluorescent Proteins: Advanced Imaging Applications and Future Design. Angew. Chem. Int. Ed. 2012, 51, 10724-10738.

(19) Andresen, M.; Stiel, A. C.; Fölling, J.; Wenzel, D.; Schönle, A.; Egner, A.; Eggeling, C.; Hell, S. W.; Jakobs, S. Photoswitchable Fluorescent Proteins Enable Monochromatic Multilabel Imaging and Dual Color Fluorescence Nanoscopy. Nat. Biotechnol. 2008, 26, 1035-1040.

(20) Faraji, S.; Krylov, A. I. On the Nature of an Extended Stokes Shift in the mPlum Fluorescent Protein. J. Phys. Chem. B 2015, 119, 13052-13062. 
(21) Amat, P.; Nifosì, R. Spectral "Fine" Tuning in Fluorescent Proteins: The Case of the GFP-Like Chromophore in the Anionic Protonation State. J. Chem. Theory Comput. 2013, 9, 497-508.

(22) Hasegawa, J.-Y.; Fujimoto, K.; Swerts, B.; Miyahara, T.; Nakatsuji, H. Excited States of GFP Chromophore and Active Site Studied by the SAC-CI Method: Effect of ProteinEnvironment and Mutations. J. Comput. Chem. 2007, 28, 2443-2452.

(23) Park, J. W.; Rhee, Y. M. Emission Shaping in Fluorescent Proteins: Role of Electrostatics and $\pi$-Stacking. Phys. Chem. Chem. Phys. 2016, 18, 3944-3955.

(24) List, N. H.; Olsen, J. M. H.; Jensen, H. J. A.; Steindal, A. H.; Kongsted, J. MolecularLevel Insight into the Spectral Tuning Mechanism of the DsRed Chromophore. J. Phys. Chem. Lett. 2012, 3, 3513-3521.

(25) Jacchetti, E.; Gabellieri, E.; Cioni, P.; Bizzarri, R.; Nifosì, R. Temperature and Pressure Effects on GFP Mutants: Explaining Spectral Changes by Molecular Dynamics Simulations and TD-DFT Calculations. Phys. Chem. Chem. Phys. 2016, 18, 1282812838.

(26) Drobizhev, M.; Callis, P. R.; Nifosì, R.; Wicks, G.; Stoltzfus, C.; Barnett, L.; Hughes, T. E.; Sullivan, P.; Rebane, A. Long- and Short-Range Electrostatic Fields in GFP Mutants: Implications for Spectral Tuning. Sci. Rep. 2015, 5, 13223.

(27) Martin, M. E.; Negri, F.; Olivucci, M. Origin, Nature, and Fate of the Fluorescent State of the Green Fluorescent Protein Chromophore at the CASPT2//CASSCF Resolution. J. Am. Chem. Soc. 2004, 126, 5452-5464.

(28) Polyakov, I. V.; Grigorenko, B. L.; Epifanovsky, E. M.; Krylov, A. I.; Nemukhin, A. V. Potential Energy Landscape of the Electronic States of the GFP Chromophore in Different Protonation Forms: Electronic Transition Energies and Conical Intersections. J. Chem. Theory Comput. 2010, 6, 2377-2387. 
(29) Moeyaert, B.; Nguyen Bich, N.; De Zitter, E.; Rocha, S.; Clays, K.; Mizuno, H.; van Meervelt, L.; Hofkens, J.; Dedecker, P. Green-to-Red Photoconvertible Dronpa Mutant for Multimodal Super-Resolution Fluorescence Microscopy. ACS Nano 2014, 8, 16641673.

(30) Zhou, X. X.; Lin, M. Z. Photoswitchable Fluorescent Proteins: Ten Years of Colorful Chemistry and Exciting Applications. Curr. Opin. Chem. Biol. 2013, 17, 682-690.

(31) Stiel, A. C.; Trowitzsch, S.; Weber, G.; Andresen, M.; Eggeling, C.; Hell, S. W.; Jakobs, S.; Wahl, M. C. 1.8 A Bright-State Structure of the Reversibly Switchable Fluorescent Protein Dronpa Guides the Generation of Fast Switching Variants. Biochem. J. 2007, 402, 35-42.

(32) Rosenbloom, A. B.; Lee, S.-H.; To, M.; Lee, A.; Shin, J. Y.; Bustamante, C. Optimized Two-Color Super Resolution Imaging of Drp1 during Mitochondrial Fission with a Slow-Switching Dronpa Variant. Proc. Natl. Acad. Sci. 2014, 111, 13093-13098.

(33) Andresen, M.; Stiel, A. C.; Trowitzsch, S.; Weber, G.; Eggeling, C.; Wahl, M. C.; Hell, S. W.; Jakobs, S. Structural Basis for Reversible Photoswitching in Dronpa. Proc. Natl. Acad. Sci. 2007, 104, 13005-13009.

(34) Brakemann, T.; Weber, G.; Andresen, M.; Groenhof, G.; Stiel, A. C.; Trowitzsch, S.; Eggeling, C.; Grubmüller, H.; Hell, S. W.; Wahl, M. C.; Jakobs, S. Molecular Basis of the Light-Driven Switching of the Photochromic Fluorescent Protein Padron. J. Biol. Chem. 2010, 285, 14603-14609.

(35) Piatkevich, K. D.; Malashkevich, V. N.; Morozova, K. S.; Nemkovich, N. A.; Almo, S. C.; Verkhusha, V. V. Extended Stokes Shift in Fluorescent Proteins: Chromophore-Protein Interactions in a Near-Infrared TagRFP675 Variant. Sci. Rep. 2013, 3, 1847 . 
(36) Yoon, E.; Konold, P. E.; Lee, J.; Joo, T.; Jimenez, R. Far-Red Emission of mPlum Fluorescent Protein Results from Excited-State Interconversion between Chromophore Hydrogen-Bonding States. J. Phys. Chem. Lett. 2016, 7, 2170-2174.

(37) Arpino, J. A. J.; Rizkallah, P. J.; Jones, D. D. Crystal Structure of Enhanced Green Fluorescent Protein to $1.35 \AA$ Resolution Reveals Alternative Conformations for Glu222. PLoS One 2012, 7.

(38) Smyrnova, D.; Zinovjev, K.; Tuñón, I.; Ceulemans, A. Thermal Isomerization Mechanism in Dronpa and Its Mutants. J. Phys. Chem. B 2016, 120, 12820-12825.

(39) Filippi, C.; Buda, F.; Guidoni, L.; Sinicropi, A. Bathochromic Shift in Green Fluorescent Protein: A Puzzle for QM/MM Approaches. J. Chem. Theory Comput. 2012, 8, $112-124$.

(40) Melaccio, F.; del Carmen Marín, M.; Valentini, A.; Montisci, F.; Rinaldi, S.; Cherubini, M.; Yang, X.; Kato, Y.; Stenrup, M.; Orozco-Gonzalez, Y.; Ferré, N.; Luk, H. L.; Kandori, H.; Olivucci, M. Towards Automatic Rhodopsin Modeling as a Tool for HighThroughput Computational Photobiology. J. Chem. Theory Comput. 2016, 6020-6034.

(41) Morozov, D.; Groenhof, G. Hydrogen Bond Fluctuations Control Photochromism in a Reversibly Photo-Switchable Fluorescent Protein. Angew. Chem. Int. Ed. Engl. 2016, $55,576-578$.

(42) Coquelle, N.; Sliwa, M.; Woodhouse, J.; Schirò, G.; Adam, V.; Aquila, A.; Barends, T. R. M.; Boutet, S.; Byrdin, M.; Carbajo, S.; De la Mora, E.; Doak, R. B.; Feliks, M.; Fieschi, F.; Foucar, L.; Guillon, V.; Hilpert, M.; Hunter, M. S.; Jakobs, S.; Koglin, J. E.; Kovacsova, G.; Lane, T. J.; Lévy, B.; Liang, M.; Nass, K.; Ridard, J.; Robinson, J. S.; Roome, C. M.; Ruckebusch, C.; Seaberg, M.; Thepaut, M.; Cammarata, M.; Demachy, I.; Field, M.; Shoeman, R. L.; Bourgeois, D.; Colletier, J.-P.; 
Schlichting, I.; Weik, M. Chromophore Twisting in the Excited State of a Photoswitchable Fluorescent Protein Captured by Time-Resolved Serial Femtosecond Crystallography. Nat Chem 2017, advance online publication.

(43) Li, X.; Chung, L. W.; Mizuno, H.; Miyawaki, A.; Morokuma, K. Primary Events of Photodynamics in Reversible Photoswitching Fluorescent Protein Dronpa. J. Phys. Chem. Lett. 2010, 1, 3328-3333.

(44) Fdez. Galvan, I.; Delcey, M. G.; Pedersen, T. B.; Aquilante, F.; Lindh, R. Analytical State-Average Complete-Active-Space Self-Consistent Field Nonadiabatic Coupling Vectors: Implementation with Density-Fitted Two-Electron Integrals and Application to Conical Intersections. J. Chem. Theory Comput. 2016, 12, 3636-3653.

(45) Park, J. W.; Shiozaki, T. Analytical Derivative Coupling for Multistate CASPT2 Theory. J. Chem. Theory Comput. 2017, 13, 2561-2570.

(46) Pronk, S.; Pall, S.; Schulz, R.; Larsson, P.; Bjelkmar, P.; Apostolov, R.; Shirts, M. R.; Smith, J. C.; Kasson, P. M.; van der Spoel, D.; Hess, B.; Lindahl, E. GROMACS 4.5: A High-Throughput and Highly Parallel Open Source Mol. Simul. Toolkit. Bioinformatics 2013, 29, 845-854.

(47) Case, D. A.; Babin, V.; Berryman, J. T.; Betz, R. M.; Cai, Q.; Cerutti, D. S.; Cheatham, T. E.; III, T. A. D.; Duke, R. E.; Gohlke, H.; Goetz, A. W.; Gusarov, S.; Homeyer, N.; Janowski, P.; Kaus, J.; Kolossváry, I.; Kovalenko, A.; Lee, T. S.; LeGrand, S.; Luchko, T.; Luo, R.; Madej, B.; Merz, K. M.; Paesani, F.; Roe, D. R.; Roitberg, A.; Sagui, C.; Salomon-Ferrer, R.; Seabra, G.; Simmerling, C. L.; Smith, W.; Swails, J.; Walker, R. C.; Wang, J.; Wolf, R. M.; Wu, X.; Kollman, P. A. AMBER 14. 2014.

(48) Smyrnova, D.; Moeyaert, B.; Michielssens, S.; Hofkens, J.; Dedecker, P.; Ceulemans, A. 
Molecular Dynamic Indicators of the Photoswitching Properties of Green Fluorescent Proteins. J. Phys. Chem. B 2015, 119, 12007-12016.

(49) Mizuno, H.; Mal, T. K.; Walchli, M.; Kikuchi, A.; Fukano, T.; Ando, R.; Jeyakanthan, J.; Taka, J.; Shiro, Y.; Ikura, M.; Miyawaki, A. Light-Dependent Regulation of Structural Flexibility in a Photochromic Fluorescent Protein. Proc. Natl. Acad. Sci. 2008, 105, 9227-9232.

(50) Regis Faro, A.; Carpentier, P.; Jonasson, G.; Pompidor, G.; Arcizet, D.; Demachy, I.; Bourgeois, D. Low-Temperature Chromophore Isomerization Reveals the Photoswitching Mechanism of the Fluorescent Protein Padron. J. Am. Chem. Soc. 2011, 133, $16362-16365$.

(51) Li, H.; Robertson, A. D.; Jensen, J. H. Very Fast Empirical Prediction and Rationalization of Protein pKa Values. Proteins: Struct., Funct., Bioinf. 2005, 61, 704-721.

(52) Ryckaert, J.-P.; Ciccotti, G.; Berendsen, H. J. C. Numerical Integration of the Cartesian Equations of Motion of a System with Constraints: Molecular Dynamics of n-Alkanes. J. Comput. Phys. 1977, 23, 327-341.

(53) Bussi, G.; Donadio, D.; Parrinello, M. Canonical Sampling through Velocity Rescaling. J Chem Phys 2007, 126, 014101.

(54) Patriksson, A.; van der Spoel, D. A Temperature Predictor for Parallel Tempering Simulations. Phys. Chem. Chem. Phys. 2008, 10, 2073-2077.

(55) Aquilante, F.; De Vico, L.; Ferré, N.; Ghigo, G.; Malmqvist, P.-A.; Neogrády, P.; Pedersen, T. B.; Pitonák, M.; Reiher, M.; Roos, B. O.; Serrano-Andrés, L.; Urban, M.; Veryazov, V.; Lindh, R. MOLCAS 7: The next Generation. J Comput Chem 2010, 31, $224-247$. 
(56) Ponder, J. W.; Richards, F. M. An Efficient Newton-like Method for Molecular Mechanics Energy Minimization of Large Molecules. J. Comput. Chem. 1987, 8, 1016-1024.

(57) Singh, U. C.; Kollman, P. A. A Combined Ab Initio Quantum Mechanical and Molecular Mechanical Method for Carrying out Simulations on Complex Molecular Systems: Applications to the $\mathrm{CH} 3 \mathrm{Cl}+\mathrm{Cl}$ - Exchange Reaction and Gas Phase Protonation of Polyethers. J. Comput. Chem. 1986, 7, 718-730.

(58) Svensson, M.; Humbel, S.; Froese, R. D. J.; Matsubara, T.; Sieber, S.; Morokuma, K. ONIOM: A Multilayered Integrated MO + MM Method for Geometry Optimizations and Single Point Energy Predictions. A Test for Diels-Alder Reactions and Pt(P(tBu)3)2 + H2 Oxidative Addition. J. Phys. Chem. 1996, 100, 19357-19363.

(59) Cornell, W. D.; Cieplak, P.; Bayly, C. I.; Gould, I. R.; Merz, K. M.; Ferguson, D. M.; Spellmeyer, D. C.; Fox, T.; Caldwell, J. W.; Kollman, P. A. A Second Generation Force Field for the Simulation of Proteins, Nucleic Acids, and Organic Molecules. J. Am. Chem. Soc. 1995, 117, 5179-5197.

(60) Ferré, N.; Ángyán, J. G. Approximate Electrostatic Interaction Operator for QM/MM Calculations. Chem. Phys. Lett. 2002, 356, 331-339. 


\section{Graphical TOC Entry}

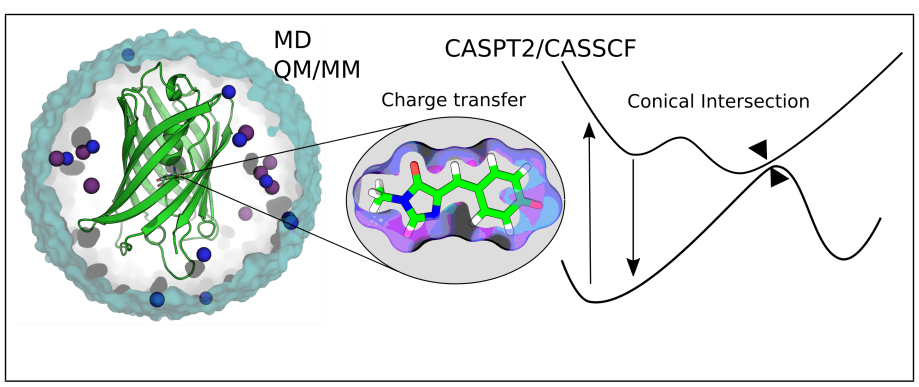

